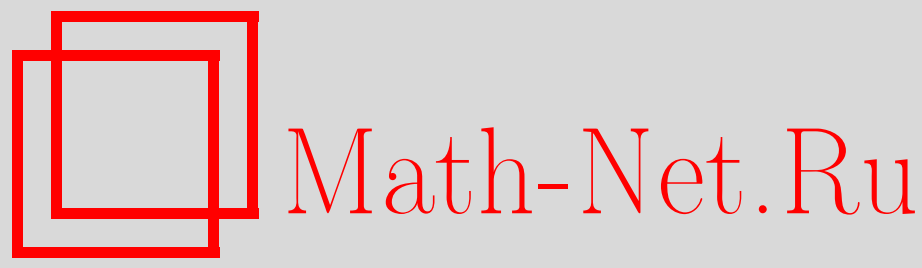

В. А. Рубаков, Могут ли галилеоны поддерживать лоренцевы кротовые норы?, ТМФ, 2016, том 187, номер 2, 338-349

DOI: https://doi.org/10.4213/tmf9070

Использование Общероссийского математического портала Math-Net.Ru подразумевает, что вы прочитали и согласны с пользовательским соглашением http://www . mathnet.ru/rus/agreement

Параметры загрузки:

IP : 3.80 .181 .102

26 апреля 2023 г., 14:32:16

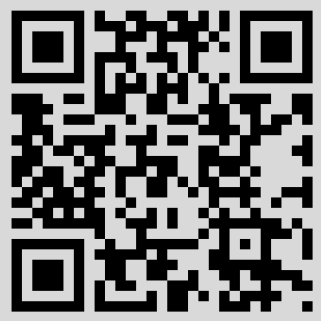




\section{МОГУТ ЛИ ГАЛИЛЕОНЫ ПОДДЕРЖКИВАТЬ ЛОРЕНЦЕВЫ КРОТОВЫЕ НОРЫ?}

Обсуждается возможность построения решений для устойчивых статических сферически-симметричных и асимптотически плоских лоренцевых кротовых нор в общей теории относительности с обобщенным полем галилеонов $\pi$. В предположении, что пространство-время Минковского получается при $\partial \pi=0$, оказывается, что имеются трудности в согласовании свойств тензора энергии-импульса, необходимых для поддержания кротовой норы (нарушение изотропного условия энергодоминантности), и условия устойчивости возмущений поля галилеонов вокруг предполагаемого решения (отсутствие духов и градиентных неустойчивостей). В трехмерном пространстве-времени эти сложности настолько существенны, что позволяют исключить кротовые норы с описанными выше свойствами. В высших размерностях, включая наиболее физически интересный случай четырехмерного пространства-времени, кротовые норы, если таковые имеются, должны иметь весьма замысловатые формы.

Ключевые слова: кротовые норы, поле галилеона.

DOI: $10.4213 /$ tmf9070

\section{1. ВВЕДЕНИЕ И СВОДКА РЕЗУЛЬТАТОВ}

Лоренцевы кротовые норы [1], если они существуют, должны представлять собой довольно любопытные объекты [2]-[5]. В классической общей теории относительности, однако, асимптотически плоские лоренцевы кротовые норы можно поддерживать только с помощью материи, которая нарушает изотропное условие энергодоминантности (ИУЭ) [3], [6], [7], в то время как известные формы материи не обладают таким свойством (интересный пример лоренцевой кротовой норы, которая не является асимптотически плоской, приведен в работе [8]). Более того, в большинстве моделей классической теории поля нарушения ИУЭ, если таковые имеются, обременены духами и/или градиентными неустойчивостями. В частности, скалярные теории поля с лагранжианами, содержащими пространственно-временные производные

Работа поддержана со стороны Российского научного фонда, грант 14-22-00161.

* Институт ядерных исследований РАН, Москва, Россия. E-mail: rubakov@minus.inr.ac.ru

${ }^{\dagger}$ Физический факультет, Московский государственный университет им. М. В. Ломоносова, Москва, Россия 


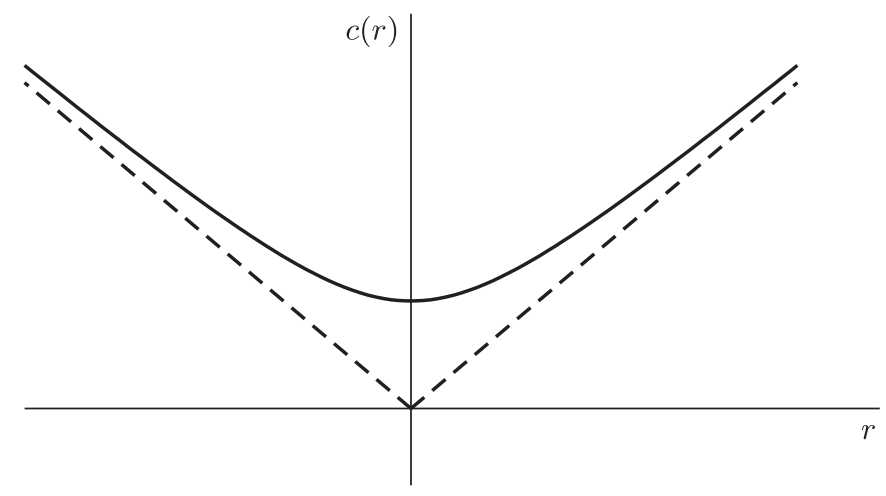

Рис. 1. Поведение метрического коэффициента $c$.

не выше первого порядка, могут допускать решения, нарушающие ИУЭ, включая лоренцевы кротовые норы (см., например, работу [9] и приведенные в ней ссылки), однако у возмущений на таком фоне имеются градиентные неустойчивости и/или духи [10].

С другой стороны, известно, что существуют скалярные теории поля, в которых лагранжианы содержат члены со второй производной, и тем не менее полевые уравнения для них являются уравнениями второго порядка [11]-[16]. Эти теории, получившие название обобщенных галилеоновых моделей, допускают классические нарушающие ИУЭ решения космологического типа, не имеющие явных патологий [17]-[20] (обзор на эту тему приведен, например, в статье [21]), если не считать проблему сверхсветовых скоростей [22], [23].

Таким образом, естественно поставить вопрос, допускают ли обобщенные галилеоновы теории существование устойчивых лоренцевых кротовых нор в рамках общей теории относительности? Настоящая работа посвящена обсуждению именно этого вопроса, хотя и не в полной общности. Уточнения таковы.

Во-первых, мы выделяем подкласс обобщенных галилеонов, для которых лагранжианы имеют наиболее изученный вид [18], [19]

$$
L=F(\pi, X)+K(\pi, X) \square \pi,
$$

где $\pi$ - поле галилеона, $F$ и $K$ - произвольные лагранжевы функции,

$$
X=\nabla_{\mu} \pi \nabla^{\mu} \pi, \quad \square \pi=\nabla_{\mu} \nabla^{\mu} \pi .
$$

Далее будем предполагать, что существует предел пространства Минковского, который имеет место при $\partial_{\mu} \pi=0$. Заметим, что если функция $K$ регулярна при $X=0$, то можно положить

$$
\left.K(\pi, X)\right|_{X=0}=0,
$$

поскольку член $K(\pi, 0) \square \pi$ можно включить в $F$ с помощью интегрирования по частям. Заметим также, что скалярный потенциал, если таковой имеется, содержится в $F$, а именно $V(\pi)=-F(\pi, 0)$.

Во-вторых, мы рассматриваем статические сферически-симметричные кротовые норы в $(d+2)$-мерном пространстве-времени. Метрика (с сигнатурой $(+,-, \ldots,-))$ 
имеет вид

$$
d s^{2}=a^{2}(r) d t^{2}-b^{2}(r) d r^{2}-c^{2}(r) \gamma_{\alpha \beta} d x^{\alpha} d x^{\beta},
$$

где $x^{\alpha}$ и $\gamma_{\alpha \beta}$ - координаты и метрика на единичной $d$-мерной сфере. Координата $r$ принимает значения от $-\infty$ до $+\infty$, а геометрия кротовой норы предполагается асимптотически плоской. При $d \geqslant 2$ (размерность пространства-времени равна четырем или более) из этого условия следует, что метрические коэффициенты имеют асимптотику (см. рис. 1)

$$
d \geqslant 2: \quad a(r) \rightarrow a_{ \pm}, \quad b(r) \rightarrow 1, \quad \frac{c(r)}{r} \rightarrow \pm 1 \quad \text { при } \quad r \rightarrow \pm \infty
$$

где $a_{ \pm}-$положительные константы.

В трехмерном пространстве-времени $(d=1)$ асимптотики коэффициента $c(r)$ не столь ограничительны,

$$
d=1: \quad a(r) \rightarrow a_{ \pm}, \quad b(r) \rightarrow 1, \quad \frac{c(r)}{r} \rightarrow \pm C_{ \pm} \quad \text { при } \quad r \rightarrow \pm \infty,
$$

где $C_{ \pm}$- положительные константы. Мы также считаем, что поле галилеона, которое, как предполагается, поддерживает кротовую нору, является статическим и сферически-симметричным, $\pi=\pi(r)$ и

$$
\pi^{\prime}(r) \rightarrow 0 \quad \text { при } \quad r \rightarrow \pm \infty,
$$

где штрих обозначает производную $d / d r$.

Наша главная забота - это устойчивость, а именно отсутствие духов и градиентных неустойчивостей в возмущениях поля галилеонов в окрестности решения $\pi(r)$. Мы увидим, что в любой размерности имеются сложности в согласовании свойств галилеонова тензора энергии-импульса, который поддерживает кротовую нору, с одной стороны, и требования устойчивости - с другой. В трехмерном пространствевремени $(d=1)$ эти сложности настолько значительны, что при очень мягких допущениях относительно асимптотического поведения производной $\pi^{\prime}$ на пространственной бесконечности можно показать, что вообще не существует устойчивых кротовых нор. Этот результат в определенной мере аналогичен результатам работы [24].

Мы не можем доказать аналогичную теорему запрета для пространства-времени размерности 4 или выше, однако мы покажем, что простейшие формы кротовых нор также противоречат условию устойчивости. Под кротовой норой простейшей формы мы понимаем решение, для которого $d c / d R$ (здесь $R=\int b d r-$ собственное радиальное расстояние) монотонно возрастает ${ }^{1)}$ от -1 до 1 с ростом $R$ от $-\infty$ до $+\infty$ (см. рис. 2).

Аналогичное свойство имеет место в системе координат ${ }^{2)}$, где $b(r)=a^{-1}(r)$. А именно, если $d c / d r$ монотонно возрастает в этой системе координат при возрастании $r$, то кротовая нора неустойчива. В этом состоит наш основной результат: при принятых выше допущениях галилеоны не могут поддерживать устойчивые кротовые норы в трехмерном пространстве-времени, в то время как в высших размерностях устойчивые поддерживаемые галилеонами кротовые норы, если они вообе существуют, должны иметь весьма нетривиальные свойства.

1) На самом деле наше наблюдение несколько сильнее: кротовые норы, для которых $|d c / d R| \leqslant 1$ при всех $R$, несовместны с условием устойчивости.

2) Такую систему координат можно называть шварцшильдовой. 


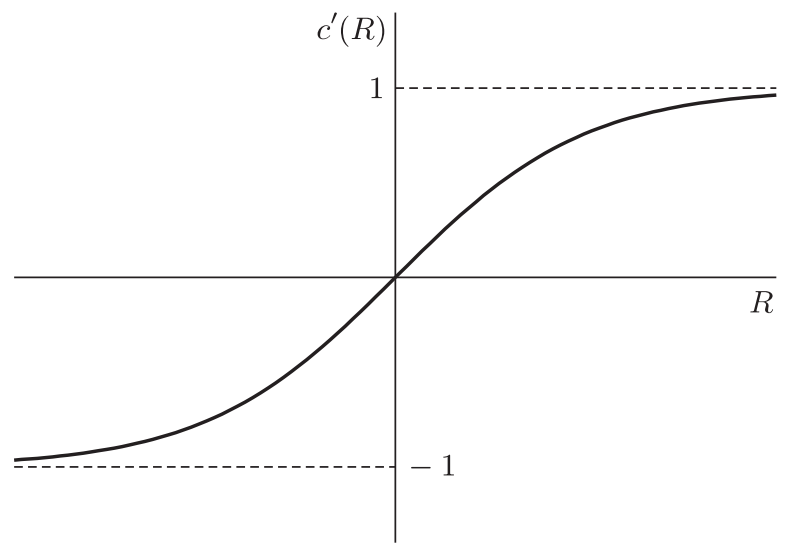

Рис. 2. Поведение производной $c^{\prime}(R)$ для кротовой норы простейшей формы (здесь $R=\int b d r-$ собственное радиальное расстояние).

Работа построена следующим образом. В разделе 2 обсуждаются свойства тензора энергии-импульса, поддерживающего кротовую нору. В разделе 3 рассмотрены статические сферически-симметричные галилеоны, представлен вид тензора энергии-импульса и выписаны условия устойчивости возмущений поля галилеонов. Основные результаты работы получены в разделе 4 , а в разделе 5 подводятся итоги.

\section{2. НАРУШЕНИЕ УСРЕДНЕННОГО ИУЭ}

Установим некоторые характерные свойства тензора энергии-импульса, поддерживающего кротовую нору. Они аналогичны свойствам, приводящим к нарушению усредненного ИУЭ (УИУЭ), см. книгу [1] и приведенную в ней литературу. Для этого напомним, что поточечное нарушение ИУЭ имеет место, когда $T_{\mu}^{\nu} k^{\mu} k_{\nu}<0$ для некоторого изотропного вектора $k^{\mu}$, в то время как нарушение УИУЭ в широком смысле представляет собой условие отрицательности значения некоторого линейного интеграла от $T_{\mu}^{\nu} k^{\mu} k_{\nu}$. В нашем контексте соответствующий изотропный вектор имеет компоненты $k^{0}=a^{-1}, k^{r}=b^{-1}$ и $k^{\alpha}=0$, так что $T_{\mu}^{\nu} k^{\mu} k_{\nu}=T_{0}^{0}-T_{r}^{r}$; линейные интегралы, которые будут встречаться в настоящей работе, имеют вид $\int_{-\infty}^{+\infty} \varphi(r)\left(T_{0}^{0}-T_{r}^{r}\right) d r$, где $\varphi(r)-$ положительная функция.

Положим $8 \pi G=1$ и используем уравнения Эйнштейна ${ }^{3)} T_{\nu}^{\mu}=G_{\nu}^{\mu}$, где

$$
\begin{aligned}
G_{0}^{0} & =d\left[\frac{c^{\prime} b^{\prime}}{c b^{3}}-\frac{c^{\prime \prime}}{c b^{2}}-\frac{d-1}{2}\left(\frac{c^{\prime 2}}{c^{2} b^{2}}-\frac{1}{c^{2}}\right)\right], \\
G_{r}^{r} & =-d\left[\frac{a^{\prime} c^{\prime}}{a c b^{2}}+\frac{d-1}{2}\left(\frac{c^{\prime 2}}{c^{2} b^{2}}-\frac{1}{c^{2}}\right)\right], \\
G_{\beta}^{\alpha} & =\delta_{\beta}^{\alpha} G^{\Omega}
\end{aligned}
$$

${ }^{3)} \mathrm{B}$ этих уравнениях $T_{0}^{0}=\rho, T_{r}^{r}=-p_{\mathrm{r}}, T_{\beta}^{\alpha}=-\delta_{\beta}^{\alpha} p_{\mathrm{t}}$, где $\rho-$ плотность энергии, $p_{\mathrm{r}}-$ радиальное давление, a $p_{\mathrm{t}}-$ касательное давление. Далее мы не будем использовать такую терминологию. 
здесь

$$
G^{\Omega}=-\left[\frac{a^{\prime \prime}}{a b^{2}}-\frac{a^{\prime} b^{\prime}}{a b^{3}}+(d-1)\left(\frac{c^{\prime \prime}}{c b^{2}}+\frac{c^{\prime} a^{\prime}}{c a b^{2}}-\frac{c^{\prime} b^{\prime}}{c b^{3}}\right)+\frac{(d-1)(d-2)}{2}\left(\frac{c^{\prime 2}}{c^{2} b^{2}}-\frac{1}{c^{2}}\right)\right] .
$$

Комбинируя уравнения (6а) и (6б), получаем

$$
T_{0}^{0}-T_{r}^{r}=-d \frac{a}{b c}\left(\frac{c^{\prime}}{a b}\right)^{\prime} .
$$

Последнее уравнение приводит к наиболее часто используемому виду нарушения УИУЭ [1]:

$$
\int_{-\infty}^{+\infty} \frac{b}{a}\left(T_{0}^{0}-T_{r}^{r}\right) d r=-d \int_{-\infty}^{+\infty} \frac{c^{\prime 2}}{a b c^{2}} d r<0
$$

(поверхностный член, появляющийся при интегрировании по частям, обращается в нуль в силу асимптотики (3) или (4)). Этот результат можно обобщить до

$$
\int_{-\infty}^{+\infty} \frac{b c^{\alpha}}{a}\left(T_{0}^{0}-T_{r}^{r}\right) d r<0 \quad \text { для всех } \quad \alpha \leqslant 1 .
$$

Такое обобщение делается немедленно при $\alpha<1$ (поверхностный член снова обращается в нуль), тогда как при $\alpha=1$ имеем

$$
\int_{-\infty}^{+\infty} \frac{b c}{a}\left(T_{0}^{0}-T_{r}^{r}\right) d r=-d\left(\frac{C_{+}}{a_{+}}+\frac{C_{-}}{a_{-}}\right)
$$

(где $C_{ \pm}=1$ при $d \geqslant 2$ ).

Как мы увидим далее, галилеонов тензор энергии-импульса удовлетворяет соотношению $T_{\beta}^{\alpha}=\delta_{\beta}^{\alpha} T^{\Omega}$, причем

$$
T^{\Omega}=T_{0}^{0}
$$

откуда следует, что $G^{\Omega}=G_{0}^{0}$, и это дает уравнение

$$
-\frac{c^{\prime \prime}}{b^{2} c}+\frac{c^{\prime} b^{\prime}}{b^{3} c}+\frac{a^{\prime \prime}}{b^{2} a}-\frac{a^{\prime} b^{\prime}}{b^{3} a}+(d-1) \frac{c^{\prime} a^{\prime}}{b^{2} c a}-(d-1)\left(\frac{c^{2}}{b^{2} c^{2}}-\frac{1}{c^{2}}\right)=0 .
$$

Воспользуемся им для вычисления выражения (7), имеем

$$
T_{0}^{0}-T_{r}^{r}=-\frac{d}{a b c^{d-2}}\left(\frac{a^{\prime} c^{d-2}}{b}\right)^{\prime}-\frac{d(d-1)}{c^{2}}\left(1-\frac{c^{\prime 2}}{b^{2}}\right) .
$$

Мы будем использовать это соотношение при $d \geqslant 2$. Предполагая, что

$$
a^{\prime}(r) r^{d-2} \rightarrow 0 \quad \text { при } \quad r \rightarrow \pm \infty
$$

запишем цепочку равенств

$$
\begin{aligned}
\int_{-\infty}^{+\infty} a b c^{d-2}\left(T_{0}^{0}-T_{r}^{r}\right) d r & =-d(d-1) \int_{-\infty}^{+\infty} a b c^{d-4}\left(1-\frac{c^{2}}{b^{2}}\right) d r= \\
& =-d(d-1) \int_{-\infty}^{+\infty} a c^{d-4}\left[1-\left(\frac{d c}{d R}\right)^{2}\right] d R
\end{aligned}
$$


где $R=\int b d r$ - собственное радиальное расстояние. Если правая часть равенства (12) отрицательна, это представляет собой еще одну форму нарушения УИУЭ. Заметим, что допущение (11) не является запрещающим: коль скоро полная масса, которую видит внешний наблюдатель, конечна, мы получаем ньютоновские асимптотики (напомним, что число пространственно-временных размерностей равно $d+2$ )

$$
a=1+O\left(r^{-(d-1)}\right)
$$

и поэтому $a^{\prime}=O\left(r^{-d}\right)$.

\section{3. СТАТИЧЕСКИЕ СФЕРИЧЕСКИ-СИММЕТРИЧНЫЕ ГАЛИЛЕОНЫ}

3.1. Тензор энергии-импульса. Возвращаясь к галилеонам, запишем тензор энергии-импульса теории с лагранжианом (1) в общем виде:

$T_{\mu \nu}=2 F_{X} \partial_{\mu} \pi \partial_{\nu} \pi+2 K_{X} \square \pi \cdot \partial_{\mu} \pi \partial_{\nu} \pi-\partial_{\mu} K \partial_{\nu} \pi-\partial_{\nu} K \partial_{\mu} \pi-g_{\mu \nu} F+g_{\mu \nu} g^{\lambda \rho} \partial_{\lambda} K \partial_{\rho} \pi$,

где $F_{\pi}=\partial F / \partial \pi, F_{X}=\partial F / \partial X$ и т. д. (штрих мы сохраним для обозначения $\left.d / d r\right)$,

$$
\partial_{\mu} K=K_{\pi} \partial_{\mu} \pi+2 K_{X} \nabla^{\lambda} \pi \nabla_{\mu} \nabla_{\lambda} \pi
$$

Из этого выражения мы немедленно заключаем, что в рассматриваемом статическом сферически-симметричном случае при $\pi=\pi(r)$ тензор энергии-импульса обладает свойством (9). В явном виде

$$
\begin{aligned}
& T_{0}^{0}=-F-K_{\pi}\left(\frac{\pi^{\prime}}{b}\right)^{2}+2\left(\frac{\pi^{\prime}}{b}\right)^{2} \frac{1}{b}\left(\frac{\pi^{\prime}}{b}\right)^{\prime} K_{X} \\
& T_{r}^{r}=-2\left(\frac{\pi^{\prime}}{b}\right)^{2} F_{X}-F+K_{\pi}\left(\frac{\pi^{\prime}}{b}\right)^{2}+2 K_{X}\left(\frac{\pi^{\prime}}{b}\right)^{3}\left(\frac{a^{\prime}}{b a}+d \frac{c^{\prime}}{b c}\right) .
\end{aligned}
$$

Нас интересует комбинация

$$
\frac{1}{2}\left(T_{0}^{0}-T_{r}^{r}\right)=\left(\frac{\pi^{\prime}}{b}\right)^{2}\left[F_{X}-K_{\pi}+K_{X} \frac{1}{b}\left(\frac{\pi^{\prime}}{b}\right)^{\prime}-K_{X} \frac{\pi^{\prime}}{b}\left(\frac{a^{\prime}}{a b}+d \frac{c^{\prime}}{c b}\right)\right],
$$

которая должна нарушать УИУЭ из раздела 2.

3.2. Условия устойчивости. Перейдем к обсуждению устойчивости возмущений поля галилеонов вблизи статического сферически-симметричного фона $\pi_{\mathrm{c}}(r)$. Запишем поле как $\pi=\pi_{\mathrm{c}}+\chi$. Нас интересуют моды с большими импульсом и частотой, поэтому мы сконцентрируемся на членах, которые содержат $\nabla_{\mu} \chi \nabla_{\nu} \chi$ в квадратичном лагранжиане или, что эквивалентно, на членах второго порядка, пропорциональных $\nabla_{\mu} \nabla_{\nu} \chi$, в линеаризованном полевом уравнении. Тонкость здесь заключается в том, что полевое уравнение для галилеонов содержит вторые производные метрики, а уравнения Эйнштейна содержат вторые производные поля галилеона [18] (см. также работу [19]), и аналогично - для линеаризованных уравнений для возмущений. Хитрость состоит в том, чтобы отыинтегрировать возмущения метрики из полевого уравнения галилеона, используя уравнения Эйнштейна [18]. 
Полное полевое уравнение галилеона имеет вид

$$
\begin{aligned}
\left(-2 F_{X}\right. & \left.+2 K_{\pi}-2 K_{X \pi} \nabla_{\mu} \pi \nabla^{\mu} \pi-2 K_{X} \square \pi\right) \square \pi+\left(-4 F_{X X}+4 K_{X \pi}\right) \nabla^{\mu} \pi \nabla^{\nu} \pi \nabla_{\mu} \nabla_{\nu} \pi- \\
& -4 K_{X X} \nabla^{\mu} \pi \nabla^{\nu} \pi \nabla_{\mu} \nabla_{\nu} \pi \square \pi+4 K_{X X} \nabla^{\nu} \pi \nabla^{\lambda} \pi \nabla_{\mu} \nabla_{\nu} \pi \nabla^{\mu} \nabla_{\lambda} \pi+ \\
& +2 K_{X} \nabla^{\mu} \nabla^{\nu} \pi \nabla_{\mu} \nabla_{\nu} \pi+2 K_{X} R_{\mu \nu} \nabla^{\mu} \pi \nabla^{\nu} \pi+\cdots=0
\end{aligned}
$$

(многоточием обозначены члены без вторых производных). В этом уравнении особенно нетривиален последний член. Линеаризованое уравнение можно записать в следующем виде (здесь и далее мы опускаем нижний индекс в обозначении $\pi_{\mathrm{c}}$ для фона галилеонов):

$$
\begin{aligned}
-2\left[F_{X}\right. & \left.+K_{X} \square \pi-K_{\pi}+\nabla_{\nu}\left(K_{X} \nabla^{\nu} \pi\right)\right] \nabla_{\mu} \nabla^{\mu} \chi- \\
& -2\left[2\left(F_{X X}+K_{X X} \square \pi\right) \nabla^{\mu} \pi \nabla^{\nu} \pi-2\left(\nabla^{\mu} K_{X}\right) \nabla^{\nu} \pi-2 K_{X} \nabla^{\mu} \nabla^{\nu} \pi\right] \nabla_{\mu} \nabla_{\nu} \chi+ \\
& +2 K_{X} R_{\mu \nu}^{(1)} \nabla^{\mu} \pi \nabla^{\nu} \pi+\cdots=0,
\end{aligned}
$$

где опущены члены без вторых производных функции $\chi$, а выражение $R_{\mu \nu}^{(1)}$ линейно по возмущениям метрики. Теперь воспользуемся уравнениями Эйнштейна

$$
R_{\mu \nu}-\frac{1}{2} g_{\mu \nu} R=T_{\mu \nu}
$$

или, эквивалентно, уравнениями

$$
R_{\mu \nu}=T_{\mu \nu}-\frac{1}{d} g_{\mu \nu} T_{\lambda}^{\lambda}
$$

линеаризуем тензор энергии-импульса и для последнего члена в уравнении (14) получим

$$
2 K_{X} R_{\mu \nu}^{(1)} \nabla^{\mu} \pi \nabla^{\nu} \pi=-2 K_{X}^{2}\left[-\frac{2(d-1)}{d} X^{2} \square \chi+4 X \nabla^{\mu} \pi \nabla^{\nu} \pi \nabla_{\mu} \nabla_{\nu} \chi\right]+\cdots
$$

Итоговое линеаризованное полевое уравнение для галилеонов получается из квадратичного лагранжиана

$$
\begin{aligned}
& L^{(2)}=\left[F_{X}+K_{X} \square \pi-K_{\pi}+\nabla_{\nu}\left(K_{X} \nabla^{\nu} \pi\right)\right] \nabla_{\mu} \chi \nabla^{\mu} \chi+ \\
& +\left[2\left(F_{X X}+K_{X X} \square \pi\right) \nabla^{\mu} \pi \nabla^{\nu} \pi-2\left(\nabla^{\mu} K_{X}\right) \nabla^{\nu} \pi-2 K_{X} \nabla^{\mu} \nabla^{\nu} \pi\right] \nabla_{\mu} \chi \nabla_{\nu} \chi+\delta L^{(2)},
\end{aligned}
$$

где $\delta L^{(2)}$ соответствует члену (15):

$$
\delta L^{(2)}=-\frac{2(d-1)}{d} K_{X}^{2} X^{2} \nabla_{\mu} \chi \nabla^{\mu} \chi+4 K_{X}^{2} X \nabla^{\mu} \pi \nabla^{\nu} \pi \nabla_{\mu} \chi \nabla_{\nu} \chi
$$

Если бы мы не полагали $8 \pi G=1$, то член $\delta L^{(2)}$ содержал бы множитель $8 \pi G$, а оставшаяся часть квадратичного лагранжиана не зависела бы от $G$.

Для нашего статического сферически-симметричного фона находим

$$
L^{(2)}=a^{-2} \widetilde{\mathcal{G}}^{00} \dot{\chi}^{2}-b^{-2} \widetilde{\mathcal{G}}^{r r}\left(\chi^{\prime}\right)^{2}-c^{-2} \widetilde{\mathcal{G}}^{\Omega} \gamma^{\alpha \beta} \partial_{\alpha} \chi \partial_{\beta} \chi
$$

где эффективная метрика равна

$$
\widetilde{\mathcal{G}}^{\mu \nu}=\mathcal{G}^{\mu \nu}+\delta \mathcal{G}^{\mu \nu} .
$$


Здесь

$$
\begin{aligned}
\mathcal{G}^{00} & =F_{X}-K_{\pi}-\frac{K_{X}^{\prime}}{b} \frac{\pi^{\prime}}{b}-2 K_{X} \frac{1}{b}\left(\frac{\pi^{\prime}}{b}\right)^{\prime}-2 d K_{X} \frac{c^{\prime}}{c b} \frac{\pi^{\prime}}{b} \\
\mathcal{G}^{\Omega}= & F_{X}-K_{\pi}-\frac{K_{X}^{\prime}}{b} \frac{\pi^{\prime}}{b}-2 K_{X} \frac{1}{b}\left(\frac{\pi^{\prime}}{b}\right)^{\prime}-2(d-1) K_{X} \frac{c^{\prime}}{c b} \frac{\pi^{\prime}}{b}-2 K_{X} \frac{a^{\prime}}{a b} \frac{\pi^{\prime}}{b} \\
\mathcal{G}^{r r}= & F_{X}-2 F_{X X}\left(\frac{\pi^{\prime}}{b}\right)^{2}-K_{\pi}+\frac{K_{X}^{\prime}}{b} \frac{\pi^{\prime}}{b}-2 K_{X} \frac{\pi^{\prime}}{b}\left(\frac{a^{\prime}}{b a}+d \frac{c^{\prime}}{b c}\right)+ \\
& +2 K_{X X}\left(\frac{\pi^{\prime}}{b}\right)^{2} \frac{1}{b}\left(\frac{\pi^{\prime}}{b}\right)^{\prime}+2 K_{X X}\left(\frac{\pi^{\prime}}{b}\right)^{3}\left(\frac{a^{\prime}}{b a}+d \frac{c^{\prime}}{b c}\right)
\end{aligned}
$$

где $K_{X}^{\prime}=d K_{X} / d r$, и

$$
\begin{aligned}
\delta \mathcal{G}^{00} & =\delta \mathcal{G}^{\Omega}=-\frac{2(d-1)}{d} K_{X}^{2}\left(\frac{\pi^{\prime}}{b}\right)^{4}, \\
\delta \mathcal{G}^{r r} & =\frac{2(d+1)}{d} K_{X}^{2}\left(\frac{\pi^{\prime}}{b}\right)^{4}
\end{aligned}
$$

Условия устойчивости для возмущений имеют вид

$$
\widetilde{\mathcal{G}}^{00}>0, \quad \widetilde{\mathcal{G}}^{r r} \geqslant 0, \quad \widetilde{\mathcal{G}}^{\Omega} \geqslant 0 .
$$

Только если эти условия выполнены, духи и/или градиентные неустойчивости отсутствуют.

Поскольку и $\delta \mathcal{G}^{00}$, и $\delta \mathcal{G}^{\Omega}$ обращаются в нуль в случае трехмерного пространствавремени $(d=1)$ и отрицательны в старших размерностях, из первого и третьего условий устойчивости в (17) следует, что

$$
\mathcal{G}^{00}>0, \quad \mathcal{G}^{\Omega}>0
$$

Как мы увидим далее, этим необходимым условиям совместно с требованием нарушения УИУЭ трудно удовлетворить.

Заметим, кстати, что по крайней мере в теориях, имеющих традиционный и устойчивый предел скалярной теории поля при $\partial \pi \rightarrow 0$, а именно предел $F \rightarrow X-V(\pi)$ $($ и $K \rightarrow 0$, см. уравнение $(2))$, вблизи этого предела неизбежно присутствует распространение со сверхсветовыми скоростями. Действительно, выбирая фон, для которого $\dot{\pi}=\partial_{\alpha} \pi=0$ в заданный момент времени, и полагая $a=b=1, c=r$, всегда можно получить, выбрав подходящий знак для $\pi^{\prime}$, неравенство

$$
\widetilde{\mathcal{G}}^{\Omega}-\widetilde{\mathcal{G}}^{00}=2 K_{X} \frac{\pi^{\prime}}{r}>0
$$

а это означает, что моды, нормальные к радиальному направлению, распространяются со сверхсветовыми скоростями. При малых $\pi^{\prime}$ такой фон устойчив, поскольку в этом режиме $\widetilde{\mathcal{G}}^{00}=\widetilde{\mathcal{G}}^{\Omega}=\widetilde{\mathcal{G}}^{r r}=F_{X}$ с точностью до малых поправок. Это наблюдение согласуется с более ранними результатами по сверхсветовому распространению галилеонов [23]. 


\section{4. НЕСОГЛАСОВАННОСТИ И ЗАПРЕТЫ}

4.1. Общие соображения. Рассмотрим, каким образом необходимые условия устойчивости (18) вступают в противоречие с нарушением УИУЭ, обсуждавшимся в разделе 2 . Для этого умножим величину $\mathcal{G}^{00}$, определенную в (16а), на $\mu(r)\left(\pi^{\prime} / b\right)^{2}$, где $\mu(r)$ - пока не определенная положительная функция, проинтегрируем по $r$ от $-\infty$ до $+\infty$ и затем проинтегрируем по частям третье слагаемое в правой части уравнения (16a), полагая (вспомним наше допущение (5)), что

$$
\pi^{\prime 3} K_{X} \mu \rightarrow 0 \quad \text { при } \quad r \rightarrow \pm \infty .
$$

Получим

$$
\begin{aligned}
& \int_{-\infty}^{+\infty} \mu(r)\left(\frac{\pi^{\prime}}{b}\right)^{2} \mathcal{G}^{00} d r= \\
& =\int_{-\infty}^{+\infty} \mu(r)\left(\frac{\pi^{\prime}}{b}\right)^{2}\left[F_{X}-K_{\pi}+K_{X} \frac{1}{b}\left(\frac{\pi^{\prime}}{b}\right)^{\prime}+K_{X} \frac{\pi^{\prime}}{b} \frac{1}{b}\left(\frac{\mu^{\prime}}{\mu}-\frac{b^{\prime}}{b}-2 d \frac{c^{\prime}}{c}\right)\right] d r>0
\end{aligned}
$$

Теперь положим $\mu=b c^{d} / a$ и увидим, что подынтегральное выражение в правой части уравнения (20) пропорционально $T_{0}^{0}-T_{r}^{r}$ (см. формулу (13)). Отсюда получаем

$$
\int_{-\infty}^{+\infty} \frac{b c^{d}}{a}\left(T_{0}^{0}-T_{r}^{r}\right) d r>0 .
$$

Это неравенство трудно согласовать с уравнением (8), хотя при $d \geqslant 2$ прямого противоречия нет.

Проводя аналогичную процедуру для $\mathcal{G}^{\Omega}$ с мерой $\mu=a b c^{d-2}$, получаем

$$
\int_{-\infty}^{+\infty} a b c^{d-2}\left(T_{0}^{0}-T_{r}^{r}\right) d r>0 .
$$

В более общем случае рассмотрим комбинацию

$$
(1-\beta) \mathcal{G}^{00}+\beta \mathcal{G}^{\Omega}>0, \quad 0 \leqslant \beta \leqslant 1,
$$

выберем меру в виде $\mu=a^{2 \beta-1} b c^{d-2 \beta}$ и получим неравенство

$$
\int_{-\infty}^{+\infty} a^{2 \beta-1} b c^{d-2 \beta}\left(T_{0}^{0}-T_{r}^{r}\right) d r>0 \quad \text { для всех } \quad 0 \leqslant \beta \leqslant 1,
$$

которое должно выполняться вместе с неравенством (8), выражающим нарушение УИУЭ. Очевидно, эти два неравенства трудно согласовать. Например, при $d=2$ (четырехмерное пространство-время) можно выбрать $\alpha=1$ в (8) и $\beta=1 / 2$ в (23), тогда получим

$$
\int_{-\infty}^{+\infty} \frac{b c}{a}\left(T_{0}^{0}-T_{r}^{r}\right) d r<0, \quad \int_{-\infty}^{+\infty} b c\left(T_{0}^{0}-T_{r}^{r}\right) d r>0 .
$$

Отсюда видно, что поддерживаемые галилеонами кротовые норы, если таковые имеются, должны быть весьма хитрыми. 
Заметим, что наше допущение (19) является очень мягким. Соответствующие меры растут при больших $|r|$ не быстрее, чем $|r|^{d}$, поэтому нужно, чтобы выполнялось условие $\pi^{\prime}=o\left(|r|^{-d / 3}\right)$. С другой стороны, если галилеон становится обыкновенным скалярным полем в пределе слабого поля, то мы имеем $\pi^{\prime} \propto|r|^{-d}$, и этого более чем достаточно. Другими словами, чтобы одновременно нарушались условие (19) и условие существования предела пространства Минковского при $\partial \pi=0$, галилеон должен быть весьма изощренным.

4.2. Трехмерное пространство-время. В случае трехмерного пространствавремени $d=1$, поэтому имеется прямое противоречие между неравенством (8) при $\alpha=1$ и неравенством (21). Таким образом, в данном случае справедлива теорема запрета, согласно которой не существует устойчивых статических сферическисимметричных кротовых нор в галилеоновых теориях с лагранжианами вида (1) и пространством Минковского в качестве предела при $\partial \pi=0$. Единственный способ обойти эту теорему заключается в нарушении свойства (19), которое имеет вид

$$
r \pi^{\prime 3} K_{X} \rightarrow 0 \quad \text { при } \quad r \rightarrow \pm \infty
$$

Однако, на наш взгляд, перспективы использовать эту лазейку не слишком обнадеживают.

4.3. Пространство-время размерности больше трех. В случае $d \geqslant 2$, который включает наиболее физически интересное четырехмерное пространство-время $(d=2)$, нет прямого противоречия между неравенством (8), выражающим нарушение УИУЭ и неравенством (23), обеспечивающим устойчивость. Тем не менее возможные формы кротовых нор при этом сильно ограничены. Чтобы это показать, сначала рассмотрим уравнения (12) и (22). Взятые вместе, они исключают из рассмотрения кротовые норы, для которых

$$
\left|\frac{d c}{d R}\right| \leqslant 1 \text { для всех } r,
$$

в том числе кротовые норы с монотонной производной $d c(R) / d R$ (см. рис. 2 ).

Еще одно ограничение следует непосредственно из уравнения (7). А именно, рассмотрим часто использующуюся ("шварцшильдову") систему координат, в которой $b(r)=a^{-1}(r)$. Функция $c^{\prime}(r)$ также не может быть монотонной в этой системе координат. Действительно, если $c^{\prime}(r)$ монотонно возрастает в этой системе координат от -1 до 1 , когда $r$ принимает значения от $-\infty$ до $+\infty$, то $c^{\prime \prime}(r)>0$, и всюду выполнено неравенство $T_{0}^{0}-T_{r}^{r}<0$. Это противоречит любому из неравенств $(23)$.

Два упомянутых выше свойства исключают из рассмотрения простейшие формы кротовых нор.

Завершая этот раздел, заметим, что не помогает и добавление стандартной материи, которая не нарушает ИУЭ. Выражающие нарушение УИУЭ неравенства из раздела 2 должны выполняться для полного тензора энергии-импульса, и, поскольку для стандартной материи $T_{0}^{0}-T_{r}^{r}>0$, эти неравенства должны быть по-прежнему справедливы для вклада галилеона в полный тензор $T_{\nu}^{\mu}$. Таким образом, наш анализ остается в силе. 


\section{5. ОБСУЖДЕНИЕ}

Даже несмотря на то, что наши результаты не являются полностью убедительными, они показывают, что построение поддерживаемых галилеонами кротовых нор должно быть достаточно изощренной задачей, если оно вообще возможно. Один из способов обойти возникающие при этом трудности мог бы состоять в отказе от нашего начального допущения, что режим пространства Минковского возникает при $\partial \pi=0$, что приводит к асимптотике (5). Это действительно возможно, если функции $F=F(X)$ и $K=K(X)$ зависят от $X=(\partial \pi)^{2}$, но не от самого поля $\pi$. Тогда линейный фон поля галилеонов, скажем $\pi=Q x^{1}$, где $Q$ - константа, подчиняется полевым уравнениям для галилеонов и имеет обращающийся в нуль тензор энергии-импульса, если $F\left(-Q^{2}\right)=0$ и $F_{X}\left(-Q^{2}\right)=0$. Это в некоторой мере аналогично случаю духового конденсата [25].

В нашем сферически-симметричном случае галилеон с асимптотиками $\pi \rightarrow \pm Q r$ при $r \rightarrow \pm \infty$ должен нарушать уравнение (19), поэтому приведенные выше аргументы не работают. Однако, в отличие от случая духового конденсата, дисперсионное соотношение для возмущений вблизи $\pi=Q x^{1}$ на квадратичном уровне для импульса и частоты имеет вид $\left(p^{1}\right)^{2}=0$. Как обсуждалось в работе [26], это проблематично с точки зрения эффективной теории поля: неизбежные члены высших порядков модифицируют дисперсионное соотношение, и оно приобретает вид

$$
\left(p^{1}\right)^{2}=A \frac{\omega^{4}}{\Lambda^{2}},
$$

где $\Lambda$ есть УФ-обрезание и $A$ в общем случае является величиной порядка единицы. Это может привести к градиентной неустойчивости с временным масштабом порядка $\Lambda^{-1}$. Тем не менее можно предположить, что множитель $A$ тонко настроен так, что является очень малой величиной, поэтому интерес представляет поиск устойчивых кротовых нор, для которых $\pi \rightarrow \pm Q r$ при $|r|$.

Благодарности. Автор благодарен М. Либанову за полезные обсуждения и Ф. Канфоре и С. Дезеру за полезную переписку. Отдельно следует поблагодарить А. Викмана, который указал на неточность в первоначальном анализе.

\section{Список литературы}

[1] M. Visser, Lorentzian Wormholes: From Einstein to Hawking, AIP Series in Computational and Applied Mathematical Physics, AIP, Woodbury, NY, 1995.

[2] M. S. Morris, K.S. Thorne, Amer. J. Phys., 56:5 (1988), 395-412.

[3] M.S. Morris, K. S. Thorne, U. Yurtsever, Phys. Rev. Lett., 61:13 (1988), 1446-1449.

[4] И. Д. Новиков, Н. С. Кардашев, А. А. Шацкий, УФН, 177:9 (2007), 1017-1022.

[5] А. А. Шацкий, И. Д. Новиков, Н. С. Кардашев, УФН, 178:5 (2008), 481-488.

[6] D. Hochberg, M. Visser, Phys. Rev. D, 56:8 (1997), 4745-4755, arXiv: gr-qc/9704082; M. Visser, D. Hochberg, "Generic wormhole throats", Internal Structure of Black Holes and Spacetime Singularities (Haifa, Israel, June 29-July 3, 1997), Annals of the Israel Physical Society, 13, eds. L. M. Burko, A. Ori, IOP, Bristol, 1997, 249-295, arXiv: gr-qc/9710001.

[7] D. Hochberg, M. Visser, Phys. Rev. D, 58:4 (1998), 044021, 14 pp., arXiv: gr-qc/9802046.

[8] E. Ayón-Beato, F. Canfora, J. Zanelli, Phys. Lett. B, 752 (2016), 201-205, arXiv: 1509.02659.

[9] C. Armendariz-Picon, Phys. Rev. D, 65:10 (2002), 104010, 10 pp., arXiv: gr-qc/0201027. 
[10] S. Dubovsky, T. Grégoire, A. Nicolis, R. Rattazzi, JHEP, 03 (2006), 025, 43 pp., arXiv: hep-th/0512260; R. V. Buniy, S.D. H. Hsu, B. M. Murray, Phys. Rev. D, 74:6 (2006), 063518, 12 pp., arXiv: hep-th/0606091.

[11] G. W. Horndeski, Internat. J. Theor. Phys., 10:6 (1974), 363-384.

[12] D. B. Fairlie, J. Govaerts, A. Morozov, Nucl. Phys. B, 373:1 (1992), 214-232, arXiv: hep-th/9110022; D. B. Fairlie, J. Govaerts, Phys. Lett. B, 281:1-2 (1992), 49-53, arXiv: hep-th/9202056; J. Math. Phys., 33:10 (1992), 3543-3566, arXiv: hep-th/9204074.

[13] M. A. Luty, M. Porrati, R. Rattazzi, JHEP, 09 (2003), 029, 22 pp., arXiv: hep-th/0303116; A. Nicolis, R. Rattazzi, JHEP, 06 (2004), 059, 29 pp., arXiv: hep-th/0404159.

[14] A. Nicolis, R. Rattazzi, E. Trincherini, Phys. Rev. D, 79:6 (2009), 064036, 21 pp., arXiv: 0811.2197.

[15] C. Deffayet, S. Deser, G. Esposito-Farèse, Phys. Rev. D, 82:6 (2010), 061501, 5 pp., arXiv: 1007.5278 .

[16] A. Padilla, V. Sivanesan, JHEP, 04 (2013), 032, arXiv: 1210.4026.

[17] P. Creminelli, A. Nicolis, E. Trincherini, JCAP, 10:11 (2010), 021, 25 pp., arXiv: 1007.0027; K. Hinterbichler, A. Joyce, J. Khoury, G. E. J. Miller, JCAP, 12:12 (2012), 030, 27 pp., arXiv: 1209.5742; P. Creminelli, K. Hinterbichler, J. Khoury, A. Nicolis, E. Trincherini, JHEP, 02 (2013), 006, 23 pp., arXiv: 1209.3768; K. Hinterbichler, A. Joyce, J. Khoury, G. E. J. Miller, Phys. Rev. Lett., 110:24 (2013), 241303, 5 pp., arXiv: 1212.3607; B. Elder, A. Joyce, J. Khoury, Phys. Rev. D, 89:4 (2014), 044027, 15 pp., arXiv: 1311.5889.

[18] C. Deffayet, O. Pujolas, I. Sawicki, A. Vikman, JCAP, 10:10 (2010), 026, 37 pp., arXiv: 1008.0048 .

[19] T. Kobayashi, M. Yamaguchi, J. Yokoyama, Phys. Rev. Lett., 105:23 (2010), 231302, 4 pp., arXiv: 1008.0603.

[20] T. Qiu, J. Evslin, Y.-F. Cai, M. Li, X. Zhang, JCAP, 11:10 (2011), 036, 25 pp., arXiv: 1108.0593; D. A. Easson, I. Sawicki, A. Vikman, JCAP, 11:11 (2011), 021, 28 pp., arXiv: 1109.1047; Y.-F. Cai, D. A. Easson, R. Brandenberger, JCAP, 12:8 (2012), 020, arXiv: 1206.2382; M. Osipov, V. Rubakov, JCAP, 13:11 (2013), 031, arXiv: 1303.1221; M. Koehn, J. L. Lehners, B. A. Ovrut, Phys. Rev. D, 90:2 (2014), 025005, 19 pp., arXiv: 1310.7577.

[21] В. А. Рубаков, УФН, 184:2 (2014), 137-152, arXiv: 1401.4024.

[22] A. Adams, N. Arkani-Hamed, S. Dubovsky, A. Nicolis, R. Rattazzi, JHEP, 10 (2006), 014, 37 pp., arXiv: hep-th/0602178; A. Nicolis, R. Rattazzi, E. Trincherini, JHEP, 05 (2010), 095; Erratum, 11 (2011), 128, arXiv: 0912.4258; d. A. Easson, I. Sawicki, A. Vikman, JCAP, 13:07 (2013), 014, arXiv: 1304.3903; C. de Rham, M. Fasiello, A. J. Tolley, Phys. Lett. B, 733 (2014), 46-51, arXiv: 1308.2702.

[23] G. L. Goon, K. Hinterbichler, M. Trodden, Phys. Rev. D, 83:8 (2011), 085015, 7 pp., arXiv: 1008.4580; P. de Fromont, C. de Rham, L. Heisenberg, A. Matas, JHEP, 07 (2013), 067, 28 pp., arXiv: 1303.0274; S. Garcia-Saenz, Phys. Rev. D, 87:10 (2013), 104012, 13 pp.; R. S. Kolevatov, Phys. Rev. D, 92:12 (2015), 123532, 8 pp., arXiv: 1508.00046.

[24] S. Deser, R. Jackiw, G. 't Hooft, Phys. Rev. Lett., 68:3 (1992), 267-269.

[25] N. Arkani-Hamed, H.-C. Cheng, M. A. Luty, S. Mukohyama, JHEP, 05 (2004), 074, 35 pp., arXiv: hep-th/0312099.

[26] S. L. Dubovsky, JHEP, 10 (2004), 076, 31 pp., arXiv: hep-th/0409124. 